\title{
Processing of radar images containing objects with significantly different radar cross-section in the onboard remote sensing complex for search and rescue operations in the Arctic Region
}

\author{
Vitaliy Garmash ${ }^{1}$, Stanislav Matveev ${ }^{2}$, Yuriy Petrov ${ }^{2,}$, Vasiliy Rogozhin ${ }^{1}$, \\ and Stanislav Rudika ${ }^{2}$ \\ ${ }^{1}$ JSC “Zaslon”, 196084 Saint-Petersburg, Russia \\ ${ }^{2}$ Baltic State Technical University "VOENMEH", 190005 Saint-Petersburg, Russia
}

\begin{abstract}
The method of adaptive nonlinear matching of dynamic ranges of radar signal and imaging device is proposed. The method is intended for use in the onboard remote sensing complexes during formation of radar images of the earth's surface. The method is aimed at increasing the visibility of small objects on the background of the underlying surface in order to simplify the procedures for their detection and identification. The computational complexity of the proposed algorithm makes it possible to use it in real time.
\end{abstract}

\section{Introduction}

Traditionally, the basis of onboard helicopter optoelectronic systems designed to support search and rescue operations are optical sensors of different spectral ranges. This is due to fact that it is the optical images of the objects of surrounding reality are most convenient for human visual perception. Recently, the most popular are tri-band optoelectronic systems, which include cameras of the visible range of the spectrum, as well as cameras of the shortwave and long-wave infrared ranges. The main drawback of optical systems is the significant influence of light and weather conditions on the quality of the generated images. Thus, in the dark, the visibility range of optical sensors is reduced. In addition, the presence of high-intensity precipitation significantly impairs separation of objects of interest even in infrared images. The complexes of search and rescue operations [1], designed to work in difficult climatic zones (such as the Arctic region) are subject to all-weather and all-season requirements. Therefore, the use of exclusively optical sensors in their composition is insufficient for the effective solution of the tasks. To overcome these shortcomings together with optical cameras, sensors of other spectral ranges, including radar, are introduced into such complexes.

\footnotetext{
* Corresponding author: petrov-i4@yandexl.ru
} 


\section{Imaging device and radar signal: dynamic ranges matching problem}

Radar cross-section (RSC) is the quantitative parameter that most determines the appearance of any object of the surrounding reality on the radar image. The greater RSC, the greater the amplitude of the electromagnetic wave reflected from the object and received by the radar antenna. RSC value of objects, the size of which is comparable to the resolution of the radar, determines their brightness against the background of the underlying surface. The appearance of large-size extended objects on the radar image is determined by the peculiarities of the spatial distribution of the RSC of its constituent elements.

However, the visibility of any item of the radar image is determined not only by local difference between the RSC of its own and the surrounding background, but also by the global distribution of the RSC of all objects within the radar field of view during forming the image. This is due to the significant difference in the range of RSC's of items of the observed scene with the dynamic range of the imaging device. Thus, the RSC of objects that may be interesting for radar detection and recognition may vary in tens, hundreds or even thousands of times. However, the dynamic range of a typical digital imaging device for monochrome video is usually limited to 256 degrees of brightness. At the same time, in the conditions of search and rescue operations, it is extremely important to display a radar image so that all these objects have a sufficient level of local contrast and can be distinguished by a human eye.

The solution of this problem is due to the need to compress the dynamic range of the received radar signal and its coordination with a fixed range of the monitor. The traditional approach is a statistical method based on the calculation of the mean $\mu$ and standard deviation $\sigma$ of the radar signal, followed by the coordination to the interval:

$$
(\mu-3 \sigma ; \mu+3 \sigma)
$$

with the range of the visualization device according to the linear law. Radar images formed with the statistical method of dynamic ranges matching are convenient for human visual perception. However, there are difficulties in distinguishing small-sized locally contrasting items, if the radar survey area includes object that have RSC several orders of magnitude larger.

To overcome these difficulties the nonlinear methods of dynamic ranges matching can be used. The most common are the logarithmic and power transformation, as well as the Retinex algorithm [2]. Logarithmic transformation establishes the relationship between the output $S o$ and input $S i$ value of the amplitude of the received signal at each point of the image with indexes $i, j$ by law:

$$
S o_{i, j}=N \log \left(S i_{i, j}\right) / \log \left(S i_{\max }\right)
$$

where $N$ is an avaliable number of degrees of brightness, and $S i_{\max }$ is maximal amplitude of the signal received during radar image formation. Power transformation converts the input signal to the output image according to the expression:

$$
S o_{i, j}=N\left(S i_{i, j} / S i_{\max }\right)^{\gamma}
$$

where $\gamma$ is a gamma parameter, which typically takes the value of 2.2.

Retinex algorithm is a vision model based on a simplified simulation of the biological structure of the visual tract of animals. The main advantage of the Retinex model is the mechanism of dynamic compression of image lighting, which allows to register bright and darkened areas of the image that are lost during the transition from the natural dynamic range to a fixed range of image representation in the computer. The compression takes place according to the formula: 


$$
S o_{i, j}=\log \left(S i_{i, j}\right)-\log \left(S i_{i, j} * G_{i, j}\right)
$$

where * is the convolution operation, $G_{i, j}$ - the smoothing Gaussian function.

The above-described methods of dynamic range compression help to increase the distinguishability of individual details in poorly lit areas of images by increasing the level of their local contrast. However, when they are used in relation to the processing of radar images, a negative effect is also observed, which manifests itself in the brightest areas of the image. It is expressed in the loss of extended objects their spatial RSC's distribution and their merger into large illuminated spots.

\section{Synthesis of the new method for dynamic ranges adaptive matching}

The methods described in the previous chapter are not able to provide an acceptable result when processing radar images of the Earth's surface. However, it can be achieved by combining them and adapting to the features of such images. In this case, the algorithm that provides the desired result must fit the following requirements:

- The application of the algorithm should increase the distinguishability of small items, locally contrasting against the background of the underlying surface;

- The application of the algorithm should not significantly worsen the local contrast within large extended objects with distributed RSC;

- The brightness of the resulting image of objects with a small RSC should not exceed the brightness of objects with a significantly larger RSC;

- According to the radar theory $[3,4]$, the histogram of the resulting image must comply with the Rayleigh distribution law.

The proposed method is based on the Retinex algorithm, which is due to its sensitivity to the characteristics of the local neighborhoods of each image area and providing the best result with increasing the visual distinguishability of small-size radio-contrast objects among all the nonlinear methods discussed in chapter 2. The Retinex algorithm is modified and combined with the logarithmic method in such a way as to neutralize the effect of deterioration of local contrast in bright areas of the image and counteract the decrease in the brightness of objects with large RSC. This allows them not to merge into large bright "spots" and increases their visibility on the surrounding background. The conversion of the received radar signal is carried out in accordance with the expression:

$$
S o_{i, j}=S i_{i, j} \cdot \log \left(S i_{i, j}\right) /\left(S i_{i, j} * G_{i, j}\right)
$$

To make the histogram of the formed radar image as close as possible to the Rayleigh view, we present the result obtained at step (5) to the range $\left(0 ; 3 \cdot \sigma_{r}\right)$ :

$$
\sigma_{r}=2 \cdot \dot{m} 2 / \pi
$$

where $\dot{m}$ is the estimation of mean of $S o_{i, j}$.

Figure 1 shows the radar image obtained by a traditional statistical approach to the coordination of the dynamic ranges of the received signal and the imaging device, and figure 2 demonstrated the image obtained using the proposed algorithm. It can be seen from the images that the application of the developed method contributed to a significant increase in the distinguishability of small objects on the background of the water surface while maintaining an acceptable level of local contrast throughout the entire image. 


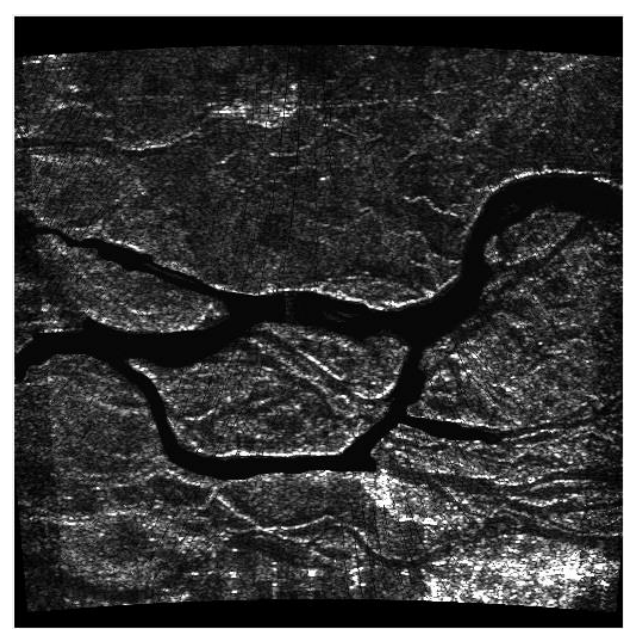

Fig. 1. Radar image of the earth's surface before non-linear processing.

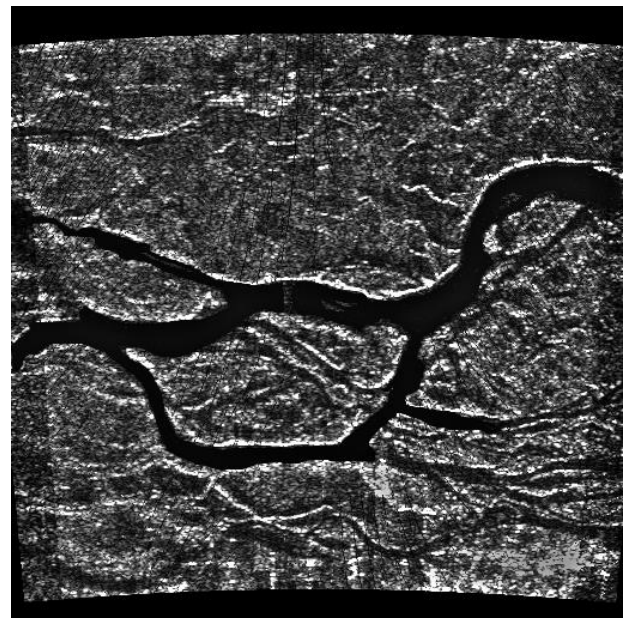

Fig. 2. Radar image of the earth's surface after non-linear processing.

\section{Conclusion}

This paper proposes the method of radar image formation, focused on providing the high level of visual distinguishability of objects that may have significantly different RSC. The method implements adaptive dynamic range matching of radar signal and imaging device, which is a combination of different types of nonlinear processing. The application of the method helps to increase the distinguishability of small-sized radio-contrast elements, while maintaining the level of local contrast within large extended objects and providing the formation of images with the brightness distribution characteristic of radar pictures. The computational complexity of the algorithm makes it possible to implement it as part of the software of embedded onboard calculators used in avionics.

The work was carried out in accordance with the decree of the Government of the Russian Federation from 09.04.2010 № 218 (PROJECT 218) in the framework of R\&D, executing with the financial support of the Ministry of education and science of the Russian Federation (agreement № 074-11-2018-025 from 13.07.2018). Work is performed in lead R\&D performer organization: Federal State Budgetary Educational Institution of Higher Professional Education BSTU "VOENMEH" named after D.F. Ustinov. 


\section{References}

1. S.A. Matveev, A.N. Bizov, S.Yu. Bistrov, V.N. Garmash, S.I. Isenko, D.M. Korobochkin, Yu.V. Petrov, S.A. Rudika, S.Yu. Strahov, A.N. Sircev. Helicopter system that provide information support for safety of flights and conduct search and rescue operations, Vestnik KRSU. 18 (9), v. 18(9), pp. 60-64 (2018)

2. E. Land, J. McCann. Lightness and Retinex Theory, Journal of the Optical Society of America, v. 61(1), pp. 1-11 (1971)

3. A. Pasmurov, J. Zinoviev, Radar Imaging and Holography. IET radar, sonar and navigation series 19 (IET, London, 2005)

4. G.S. Kondratenkov, A.Y. Frolov, Radiovidenie. Radiolokatsionnye sistemy distantsionnogo zondirovaniya Zemli. Uchebnoe posobie dlya vuzov. (Radiotehnika, Moscow, 2005). 